\title{
Comparison of Numerical Schemes for a Realistic Computational Aeroacoustics Benchmark Problem
}

\author{
R. Hixon and J. Wu \\ Mechanical, Industrial, and Manufacturing Engineering Department \\ University of Toledo \\ Toledo, $\mathrm{OH} 43606$ \\ M. Nallasamy \\ QSS Group, Inc. \\ NASA Glenn Research Center \\ Cleveland, $\mathrm{OH} 44135$ \\ S. Sawyer \\ Mechanical Engineering Department \\ The University of Akron \\ Akron, $\mathrm{OH} 44325$ \\ R. Dyson \\ NASA Glenn Research Center \\ Cleveland, $\mathrm{OH} 44135$
}

\begin{abstract}
In this work, a nonlinear structured-multiblock CAA solver, the NASA GRC BASS code, will be tested on a realistic CAA benchmark problem. The purpose of this test is to ascertain what effect the high-accuracy solution methods used in CAA have on a realistic test problem, where both the mean flow and the unsteady waves are simultaneously computed on a fully curvilinear grid from a commercial grid generator. The proposed test will compare the solutions obtained using several finite-difference methods on identical grids to determine whether high-accuracy schemes have advantages for this benchmark problem.
\end{abstract}

\section{Introduction}

The field of Computational Aeroacoustics (CAA) is concerned with the time-accurate calculation of unsteady flow fields. In order to accurately propagate the unsteady acoustic, vortical, and entropy waves, high-accuracy numerical differencing schemes have been developed which require very few grid points per disturbance wavelength to calculate an accurate value of the spatial derivative (see Refs. 1 and 2 for an overview of CAA developments). These schemes have been extended for use in nonlinear flow calculations, and have produced very good results (e.g., Refs. 3-5).

However, for realistic flow calculations using curvilinear grids, it is not clear if these high-accuracy schemes retain the advantages that they show for model problems. 
Previous work has indicated that the grid generator has an effect on the attainable accuracy of a numerical scheme ${ }^{6}$, even with a very smooth grid from a commercial grid generator. $^{7}$

In the proposed work, the NASA BASS code will be applied to the CAA Benchmark problem of a vortical gust impinging on a loaded $2 \mathrm{D}$ cascade. ${ }^{8}$ The BASS code has four spatial differencing options: explicit $2^{\text {nd }}$ order, explicit $6^{\text {th }}$ order, optimized DRP ${ }^{9}$, and prefactored compact $6^{\text {th }}$ order. $^{10}$ While it is expected that the three high-accuracy schemes will perform adequately, the question is whether they will perform better than the low-order scheme on a realistic problem.

It must be noted at this point that this test problem may well be weighted in favor of the $2^{\text {nd }}$ order explicit scheme because the wavelength of the vortical gust is very long and the computational boundaries are very close. Thus, if the high-accuracy schemes provide a measurably better answer, this will be a strong indication that high-accuracy schemes are useful for traditional CFD problems as well as CAA.

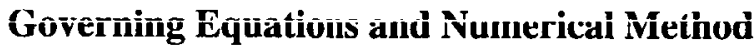

In this work, the Euler equations are solved. The 2D nonlinear Euler equations may be written in Cartesian form as:

$$
Q_{t}+E_{x}+F_{y}=0
$$

The NASA Glenn Research Center BASS code was used to solve this equation..$^{4-6,11-12}$ The BASS code uses optimized explicit time marching combined with high-accuracy finite-differences to accurately compute the unsteady flow. The code is parallel, and uses a block-structured curvilinear grid to represent the physical flow domain. A constantcoefficient $10^{\text {th }}$ order artificial dissipation model ${ }^{13}$ is used to remove unresolved highfrequency modes from the computed solution.

The BASS code solves the Euler equations using the nonconservative chain-rule formulation; previous experience has indicated that the formal lack of conservation is offset by the increased accuracy of the transformed equations..$^{3-5}$ The chain-rule form of the Euler equations are:

$$
\begin{gathered}
Q_{t}+\xi_{t} Q_{\xi}+\eta_{t} Q_{\eta} \\
+\xi_{x} E_{\xi}+\eta_{x} E_{\eta} \\
+\xi_{y} F_{\xi}+\eta_{y} F_{\eta}=0
\end{gathered}
$$

For this work, the optimized low-storage RK56 scheme of Stanescu and Habashi ${ }^{14}$ was combined with the prefactored sixth-order compact differencing scheme of Hixon ${ }^{10}$. 


\section{Proposed Work}

In this work, the CAA benchmark cascade problem given in Ref. 8 will be computed using the NASA GRC BASS code. The BASS code will be run using various spatial differencing schemes of different accuracies, and the results will be compared to determine the effectiveness of the high-accuracy finite-difference schemes currently used in CAA codes on a realistic test problem. The grid density and stretching will also be varied to investigate the grid density required for an accurate solution.

\section{References}

1) Tam, C. K. W., 'Computational Aeroacoustics: Issues and Methods', AIAA Journal, Vol. 33, No. 10, 1995, p. 1788-1796.

2) Lele, S. K., 'Computational Aeroacoustics: A Review', AIAA Paper 97-0018, January 1997.

3) Hixon, R., Mankbadi, R. R., and Scott, J. R., 'Validation of a High-Order Prefactored Compact Code on Nonlinear Flows with Complex Geometries', AIAA Paper 20011103, Jan. 2001.

4) Nallasamy, M., Hixon, R., Sawyer, S., Dyson, R., and Koch, L., 'A Parallel Simulation of Rotor Wake - Stator Interaction Noise', AIAA Paper 2003-3134, May 2003.

5) Sawyer, S., Nallasamy, M., Hixon, R., Dyson, R., and Koch, D., 'Computational Aeroacoustic Prediction of Discrete-Frequency Noise Generated by a Rotor-Stator Interaction', AIAA Paper 2003-3268, May 2003.

6) Hixon, R., Nallasamy, M., and Sawyer, S., 'Effect of Grid Singularities on the Solution Accuracy of a CAA Code', AIAA Paper 2003-0879, Jan. 2003.

7) GridPro/az3000, Program Development Corporation, White Plains, NY.

8) www.math.fsu.edu/caa4

9) Tam, C. K. W. and Webb, J. C., 'Dispersion-Relation-Preserving Finite-Difference Schemes for Computational Acoustics', Journal of Computational Physics, Vol. 107, 1993, pp. 262-281

10)Hixon, R., 'Prefactored Small-Stencil Compact Schemes', Journal of Computational Physics, Vol. 165, 2000, p. 522-541.

11)Hixon, R, Nallasamy, M., and Sawyer, S., 'Parallelization Strategy for an Explicit Computational Aeroacoustics Code', AIAA Paper 2002-2583, July 2002.

12)Hixon, R., Nallasamy, M., Sawyer, S., and Dyson, R., 'Mean Flow Boundary Conditions for Computational Aeroacoustics', AIAA Paper 2003-3299, May 2003.

13)Kennedy, C. A. and Carpenter, M. H., 'Several New Numerical Methods for Compressible Shear-Layer Simulations', Applied Numerical Mathematics, Vol. 14, 1994, pp. 397-433.

14)Stanescu, D. and Habashi, W. G., '2N-Storage Low Dissipation and Dispersion Runge- Kutta Schemes for Computational Acoustics', Journal of Computational Physics, Vol. 143, No. 2, 1998, p. 674-681. 